Thorax (1974), 29, 349.

\title{
Cervical lung hernia
}

\author{
R O B IN G. LIGHTW O O D a d W. P. CLELAND \\ The Brompton and Hammersmith Hospitals, London
}

\begin{abstract}
Lightwood, R. G., and Cleland, W. P. (1974). Thorax, 29, 349-351. Cervical lung hernia. Lung hernias occur in the cervical position in about one third of cases. The remainder appear through the chest wall. Some lung hernias are congenital, but trauma is the most common cause. The indications for surgery depend upon the severity of symptoms. Repair by direct suture can be used for small tears in Sibson's (costovertebral) fascia while larger defects have been closed using prosthetic materials. Four patients with cervical lung hernia are described together with an account of their operations.
\end{abstract}

Lung hernia is a rare condition in which part of the lung protrudes through a defect in the normal boundaries of the thoracic cavity. Such hernias occur congenitally when the parietal pleura remains intact, or they may be acquired usually as the result of trauma when the parietal pleura has been disrupted.

Morel-Lavallée (1845) made an anatomical classification of lung hernias into the cervical, chest wall, and diaphragmatic types. Of the reported cases, about two-thirds have been through the chest wall following severe trauma such as occurs in war injuries (Munnell, 1968). Most of these hernias are through the anterior chest wall adjacent to the sternum and they have been repaired using autologous tissues such as periosteal rib flaps or fascia lata or with various prosthetic materials (Naclerio, 1957).

Cervical lung hernia is the less common type and results from a defect in Sibson's (costovertebral) fascia, which allows the lung to push up between the anterior scalene and sternomastoid muscles. The four cases described here belong to this group.

A third type of lung hernia is represented by a unique report (Beale, 1882) of a hernia through the diaphragm.

\section{CASE REPORTS}

CASE 1 A 30-year-old army officer was thrown from his horse in 1962. Six weeks later he noticed a swelling at the root of the neck on the right side when he coughed or strained and this gradually enlarged and then spread to the left side causing considerable discomfort. When he was examined, a swelling was present which could be felt up to the level of the thy- roid cartilage on the right and after coughing or forced expiration it also bulged across to the left side of the neck. The diagnosis was made of a lung hernia through Sibson's fascia extending across to the left side behind the oesophagus.

At right thoracotomy in 1964 there was an obvious pleural sac extending into the root of the neck behind the subclavian vessels through a linear tear in Sibson's fascia. This was closed with interrupted linen sutures after the pleural sac had been transfixed and ligated.

When last seen in 1965 he was almost symptom free and there was no demonstrable swelling on coughing or forced expiration against a closed glottis.

CASE 2 A 48-year-old woman went to Spain in an unpressurized aircraft in 1956. On landing she noticed a large painless swelling on the left side of the neck which subsided during the next three days. The swelling recurred periodically, usually after strenuous exercise, and each time it took several days to go down.

At left thoracotomy in 1957 a defect was found in Sibson's fascia leading into a recess above the first rib some 3 to $4 \mathrm{~cm}$ in diameter. After the pleura had been stripped this defect was plugged with a block of vinyl alcohol (Ivalon) sponge which was cut to a collar-stud shape, the stud fitting up into the defect, and then sutured in position.

Postoperative recovery was good and she remained trouble-free for 10 years before developing brachial neuralgia on the same side, which became severe enough to warrant a second operation two years later. The brachial plexus was exposed through a cervical incision and was freed from a dense fibrous reaction around the Ivalon sponge, the upper part of which was removed.

Following this operation there was no further pain and the hernia has not recurred after two years. 
CASE 3 A 53-year-old woman presented in 1949 complaining of a painless swelling on the right side of the neck, which usually came up overnight and then gradually subsided during the next 24 hours. As the swelling went down she expectorated large amounts of thick viscid sputum. There was no previous history to suggest a cause. A tense swelling about the size and shape of half an orange was felt above the right clavicle, deep to the scalene and sternomastoid muscles. It was resonant on percussion and had a definite cough impulse and normal breath sounds could be heard over it. The hernia was not tender and could be reduced in size by firm pressure. Surgical treatment was advised when the attacks became more frequent and prolonged with severe pain, on one occasion lasting several days.

At right thoracotomy there was a pleural sac in the angle between the internal mammary artery and the innominate vein which extended into the neck for at least $4 \mathrm{~cm}$. This was plugged with a square of tantalum gauze, which was sutured in place.

She had no further trouble from the hernia before her death from coronary thrombosis in 1970 .

CASE 4 A 65-year-old woman was seen in 1970 with a four-year history of intermittent swelling on the right side of the neck, which became more prominent on exertion but was otherwise asymptomatic. There was no past history of a precipitating cause. After forced expiration against a closed glottis a diffuse swelling, $5 \mathrm{~cm}$ in diameter, appeared medially in the right supraclavicular fossa. There was also a similar but smaller swelling on the other side. At right thoracotomy the pleura was found to be lax in an area bounded by the subclavian artery in front and the first thoracic nerve behind where the pleura could be invaginated upwards for several centimetres. The pleura was reflected and the defect in Sibson's fascia packed with Surgicel and then closed with a square of Marlex mesh, which was sutured in place.

Eight months after the operation she still had some swelling on the right side of the neck on forced expiration, but less than preoperatively, and there was still a similar but smaller bulge on the left side.

\section{DISCUSSION}

The four cases described illustrate the typical features of cervical lung hernia and there was no clinical nor radiological evidence to suggest spontaneous surgical emphysema in these patients. Case 3 has been described previously (Rigden, 1955). The radiological findings were not helpful in making a diagnosis but no films were taken during forced expiration against a closed glottis, as was advocated by Reinhart and Hermel (1951).
In each patient their symptoms were severe enough for a thoracotomy to be thought justified. Conservative treatment by external support would be extremely difficult to apply in cervical hernias, although such methods have been used for chest wall hernias (Sauerbruch and O'Shaughnessy, 1937).

The technique of operation is based upon the principles used in the repair of hernias elsewhere. Exposure must allow the margins of the defect to be freed from adherent lung or pleura before the hernia can be reduced. The transthoracic route provides such exposure and facilitates reduction of the lung. The alternative cervical route has been used by some surgeons and may be necessary in a few cases which are unsuitable for thoracotomy.

The method of repair depends upon the type of defect. Direct suture is sometimes possible as in the first case. Larger defects need more elaborate methods. The use of local structures is impracticable unlike chest wall hernias where the ribs and their periosteum are available (Blades, 1966). Instead, one of the various prosthetic materials can be adapted as required for each hernia, as was done in the last three patients. The material used should be inert to avoid the subsequent development of a fibrous reaction which involved the brachial plexus in the second case.

The results of repair are satisfactory provided that selection is restricted to those cases with a definite hernia. Not infrequently patients with chronic bronchitis and emphysema present because they have noticed supraclavicular swellings on coughing or straining (Fenichel and Epstein, 1955). These are due to a generalized weakness of Sibson's fascia and in the absence of a localized defect repair is likely to be unsatisfactory. Fortunately, operation can usually be avoided in these cases in which symptoms are seldom severe.

\section{REFERENCES}

Beale, E. C. (1882). On a case of hernia of lung through the diaphragm. Lancet, 1, 139.

Blades, B. (1966). Surgical Diseases of the Chest. 2nd edition, pp. 87-89. C. V. Mosby, St. Louis, U.S.A.

Fenichel, N. M. and Epstein, B. S. (1955). Pulmonary apical herniations. Archives of Internal Medicine, 96, 747.

Morel-Lavallée. A. (1845). Hernies du poumon. Bulletins et Mémoires de la Sociéte de Chirurgie de Paris, 1, 75.

Munnell, E. R. (1968). Herniation of the lung. Annals of Thoracic Surgery, 5, 204. 
Naclerio, E. A. (1957). Hernia of lung. Bronchopulmonary Diseases, edited by E. A. Naclerio, p. 328. Cassell, London.

Reinhart, H. A. and Hermel, M. B. (1951). Herniation of the lung in the cervical region. Radiology, 57, 204.
Rigden, B. G. (1955). Cervical lung hernia. Report of a case. Lancet, $2,803$.

Sauerbruch, F. and O'Shaughnessy, L. (1937). Thoracic Surgery, p. 167. Arnold, London.

Requests for reprints to: W. P. Cleland, F.R.C.S., The Brompton Hospital, London SW3. 